\title{
Safety and Effectiveness of Transvaginal Mesh Placement in Treating Pelvic Organ Prolapse
}

\section{Zeyu Chen}

Xuzhou Medical University

Wensheng Du

Xuzhou Medical College Affiliated Hospital

Haitao Zhu ( $0569659980 @ q q . c o m$ )

Xuzhou Medical College Affiliated Hospital

\section{Research article}

Keywords: Pelvic organ prolapse, Complication, POP-Q

Posted Date: November 13th, 2019

DOI: https://doi.org/10.21203/rs.2.17251/v1

License: (c) (1) This work is licensed under a Creative Commons Attribution 4.0 International License. Read Full License 


\section{Abstract}

To observe the clinical effect and complications of transvaginal mesh (TVM) placement on treating pelvic organ prolapse (POP).Methods From May 2014 to June 2017, 89 patients with moderate to severe pelvic organ prolapse and pelvic floor defect (PFD) had received TVM pelvic reconstruction at our hospital. The operation time, intraoperative blood loss and intraoperative complications of all patients were recorded, and the incidence of postoperative complications was also observed. In addition, the POPQ was utilized to evaluate the severity of POP in patients before surgery, as well as at 6 weeks, 6 months, 12 months and 24 months after surgery, respectively; besides, the Pelvic Floor Influence Questionnaire-7 (PFIQ-7), Pelvic Floor Dysfunction Inventory 20 (PFDI-20), and Patient Global Impression of Improvement (PGI-I) were adopted to evaluate the quality of life of patients. Results All the 89 cases had successfully completed the surgery, with the operation time of $60.0 \pm 13.0 \mathrm{~min}$ and the intraoperative blood loss of $57.2 \pm 21.9 \mathrm{~mL}$. All patients had no bladder or rectal injury during the surgery. 2 patients displayed asymptomatic recurrence , 10 cases had postoperative fever, while 3 had mesh exposure, and all of them were improved after symptomatic treatment. Upon discharge, the lumbosacral and pubic discomfort, as well as pelvic organ dragging sensation had disappeared in all patients. In the final follow-up, the PFIQ-7 and PFDI-20 scores in the 89 cases were reduced compared with those before surgery (Ps<0.05).Conclusions TVM placement is effective on treating moderate to severe POP, which is associated with a lower complication rate and higher postoperative satisfaction.

\section{Background}

Pelvic floor dysfunction is a disease that severely affects the physical and mental health of middle-aged and old-aged women. Data suggest that, the risk of incidence is as high as $50 \%$ in women that have given birth to a baby ${ }^{1}$. At present, transvaginal mesh (TVM) placement is an effective surgical procedure with little trauma for pelvic organ prolapse (POP), which has been extensively applied in moderate and severe patients ${ }^{2,3}$. However, TVM placement is also associated with the complications such as mesh corrosion/exposure, dysuresia, pelvic and vaginal pain, and dyspareunia ${ }^{4}$. As a result, the FDA has raised two safety warnings regarding the TVM placement-induced complications in 2008 and 2011. Recently, the FDA has issued a command in 2016 to re-classify the TVM used for POP from class II (special control) to class III (pre-market approval) ${ }^{5}$. Notably, these warnings and reclassification have highlighted the potential severe risks of TVM-related complications.

This study aimed to analyze the safety and effectiveness of TVM in treating moderate and severe POP at our hospital, particularly in postoperative recurrence rate, complications and patient subject satisfaction.

\section{Methods}

From December 2014 to June 2017, the clinical data, as well as mid- and long-term follow-up data of patients with POP treated with TVM placement at the Affiliated Hospital of Xuzhou Medical University were systemically reviewed. Typically, women that had given birth to a baby or the postmenopausal 


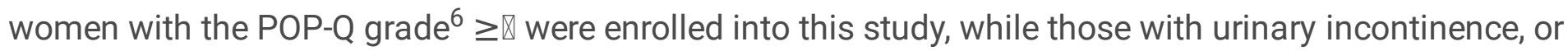
those undergoing hysterectomy or prior TVM placement were excluded from this study. The basic information of all patients is presented in Table 1. The meshes used in the surgery were Avaulta ${ }^{\circledR}$ (Bard,Murry Hill,NJ,USA) and POP-UP System $\circledast$ (Prestige Medical,Jungwon-gu Seongnam-si,Gyeonggido,KOREAN), which was selected based on the economic condition of patients. The previously reported technique was adopted in the surgical procedure ${ }^{7,8}$; at the same time, the operation time, intraoperative blood loss, and intraoperative complications of all patients were recorded to evaluate the operation safety. Afterwards, patients were followed up at 6 weeks, 6 months, 12 months and 24 months after surgery, of them, 83 cases had completed the 2-year follow-up, while 4 were lost to follow-up after 1-year of follow-up, and the remaining 2 were lost after 6 months of follow-up. The objective manifestation of healing was the POP-Q grade of $\leq \mathrm{I}$ in the last follow-up in which patients were asked to conduct the Valsalva manoeuvre in standing position. Moreover, the postoperative complications in patients were followed up by means of clinic physical examination and, Typically, the questionnaires used in this study were the Chinese version of PFDI-20 and PFIQ-7 9,10,11, among which, PFDI included the POP distress inventory 6 (POPDI-6), the Colorectal-anal distress inventory 8 (CRADI-8) and the Urinary distress inventory 6 (UDI-6); while Patient global impression of improvement (PGI-I) ${ }^{12}$ was utilized to quantity the subjective patient symptoms after surgery. PGI-I is single question of a 7-point Likert scale, which asks the patients to compare their illness at the time of answering the question with their feeling before surgery, and it ranks from 1 point (extremely good) to 7 points (extremely bad). Additionally, PGI-I was also adopted in patients developing a second prolapse or those requiring a second operation due to severe complications, so as to compare the effects before and after surgery.

The SPSS ${ }^{\circledR} 20.0$ (IBM Corporation,Armonk,NY,USA) was used for statistical analyses. Results of qualitative variables were expressed as percentage, while results of quantitative variables were expressed as means and standard deviation. The quantitative variables were compared using independent sample or paired sample t-test, while chi-squared test was adopted to test the qualitative variables. A P-value $<0.05$ was deemed as statistically significant.

\section{Results}

The average age of the 89 POP patients receiving TVM was $65.7 \pm 8.2$ years, with the average parity of $2.2 \pm 1.1$ times. Meanwhile, 83 of them $(93 \%)$ had completed the 24 -month postoperative follow-up, while only 4 (4.5\%) were followed up for 12 months, and the remaining $2(2.2 \%)$ were only followed up for 6 months due to personal reasons. As shown in Table 1, the basic conditions of patients selecting the Avaulta and the Pop-up meshes for operation were similar, and no statistically significant difference was observed ( $P>0.05)$. Similarly, preoperative POP-Q grade, as well as PFDI-20 and PFIQ-7 scores, between two mesh groups was quite close $(P>0.05)$, and a vast majority of cases had anterior prolaps $(95.5 \%)$.

In the last follow-up, 3 patients displayed asymptomatic recurrence, while 9 cases (10.1\%) required a second treatment after surgery. The average operation time was $60.0 \pm 13.0 \mathrm{~min}$, the intraoperative blood loss was $57.2 \pm 21.9 \mathrm{ml}$, the average length of hospital stay was $8.4 \pm 3.5$ days, and no obvious difference 
was detected in the above data between two mesh groups $(P>0.05$, Table 2$)$. No bladder or intestinal tract injury was observed in all patients intraoperatively and postoperatively; Two patients had anterior wall recurrence at 12 months and 1 had posterior wall recurrence at 24 months, and all were in the Avaulta group. Due to their old age and quality of life improved after surgery,the patients with recurrence did not choose further surgery.16 (18\%) of the enrolled patients had developed TVM placement-related complications, and 3 had mesh exposure at 6 months, 2 of them with obvious symptoms were treated through surgery (removal of the exposed mesh), while the other 1 with no obvious symptom was given topical application of estrogen ointment for conservative treatment. 2 patients had urinary incontinence manifestation at 6 weeks after surgery, and the symptoms were gentle, which required no further treatment. During the postoperative hospital stay, 3 patients had delayed wound healing and 9 had urinary tract infection, and no further treatment was required after the application of antibiotics and nutrition.

There were no statistical differences in the preoperative PFDI and PFIQ scores between two mesh groups (Table 1). At the last follow-up, the PFDI and PFIQ scores in all patients were markedly improved compared with those before surgery $(P<0.005)$, and PFDI had reached the least significant difference of 45 points $^{13}$. In addition, the changes in postoperative score between two groups were not statistically significant (Table 3), and both groups had attained favorable mid-term prognosis, with the average postoperative PGI-I score of $1.31 \pm 0.59$. Importantly, patients had extremely high self-evaluation satisfaction on the operation, among them, $73 \%$ suggested that their symptoms were greatly improved (PGI-I of 1), and $100 \%$ had attained overall improvement (PGI-I of 1-3). It was suggested that the symptoms were remarkably improved compared with those before surgery.

\section{Discussion}

Various surgical procedures are available for pelvic floor reconstruction in women, including the transvaginal approach, laparotomy, laparoscopic or robot-assisted approach. Among them, the transvaginal approach is associated with short operation time, length of stay and recovery time ${ }^{14,15}$. The synthesized mesh has attracted high attention and dispute in recent years, but it is the reconstruction technique that best corresponds with anatomy in pelvic floor surgery, since the mesh shape accords with the human pelvic floor. In addition, the application of mesh in pelvic floor reconstruction also conforms to the findings by Gutman $\mathrm{R}$ et al. in literature that the uterine preservation technique is the feasible surgical treatment option for prolapse of uterus, because it can best preserve the original anatomical structure and statics of the pelvic floor ${ }^{16}$.

In a study that applies TVM placement to treat POP, a conclusion is drawn through two sets of parallel, multi-center randomized controlled trials that, neither mesh or transplant material can great improve the postoperative effect, quality of life and adverse reaction within a short period of time; at the same time, over $1 / 10$ patients have developed mesh placement-related complications ${ }^{17}$. However, that research includes not only the POP patients, but also patients with urinary incontinence. By contrast, only patients with POP alone were enrolled in this study, which could eliminate the selection bias to some extent. 
Mesh exposure and invasion are the most common complications after TVM placement. According to previous literature report, the mesh exposure rate ranges from $1.4 \%$ to $36 \% 18,19,21,22$, while in the Cochrane review ${ }^{1}$, the mesh exposure rate is $12 \%$, and $8 \%$ patients require further surgical treatment. In our study, only $2(2.2 \%)$ patients had asymptomatic recurrence during postoperative follow-up ,but no further treatment was selected owing to little impact on quality of life. Another $3(3.6 \%)$ patients had mesh exposure, all of them were diabetics whose blood glucose could not be well controlled after surgery; among them, 2 patients had obvious symptoms, who had improved after surgical removal of the exposed mesh and topical application of estrogen ointment. It is generally believed that, the incidence of mesh exposure is related to the thin vagina mucosa separation, excessive tension, infection, vaginal wall atrophy, and early sexual life. Similarly, some research indicates that, diabetes will increase the mesh exposure rate by 7 folds; besides, the anterior vaginal wall is subject to a higher risk of mesh exposure than the posterior wall ${ }^{23}$. Therefore, internal medicine complications such as diabetes and hypertension were strictly controlled before surgery in this study, and the internal medicine diseases were positively controlled during the perioperative period, which were of great significance to the expected surgical wound healing, prevention of wound infection and reduction of mesh exposure rate. The sugrical procedure of Avaulta and Pop-Up has no significant difference. In the design of the puncture needle, Avaulta only uses the wire lasso connection method, while the application of Pop-Up is lock connection which was easier in practice. There is a plastic film covering outside the Pop-Up. Although it can reduce the tissue injury during the puncture process, there is no conspicuous difference in postoperative recurrence and complications.

Postoperative dyspareunia is a symptom affected by multiple factors. Typically, any transvaginal surgical procedure, regardless of the use of mesh or not, will lead to vaginal scar formation, which will subsequently result in loss of elasticity and vaginal malformation, thereby causing dyspareunia. According to the Cochrane review, the incidence of dyspareunia after surgery is $4-5 \%$, while the use of mesh will make little difference, which may be related to the vaginal approach ${ }^{1}$. In this study, only 2 $(2.2 \%)$ patients had postoperative dyspareunia, which was suggested to be related to early sexual life of patients.

The experience, skills and number of operation of the surgeon are the key factors determining whether the operation will success. As proved by Kelly ${ }^{24}$ et al., about 5\% POP women undergoing TVM placement required a second operation within 10 years to treat the mesh complications. For surgeons that carry out at least 14 operations annually, the risk of a second operation can be minimized ${ }^{24}$. Nonetheless, it should be pointed out that, surgical technique plays a crucial role in mesh corrosion, since the incidence of mesh corrosion varies from studies. For instance, a multi-center randomized controlled trial about TVM suggested that, the incidence of mesh exposure varied from $0 \%$ to $100 \%^{3}$. In order to avoid complications and reduce the risk of recurrence, we summarized some key points during TVM. Hydrodissection beneath vaginal adventitia is crucial to preserve the capillary network within the vaginal adventitia and for well separating the anterior wall of the vagina from the bladder and ureter, thereby reducing the risk of bladder or uteter damage. At the same time, attention should be paid to the flat 
placement of the mesh to avoid the formation of folds, otherwise it is easy to form a local cavity causing infection; and we use normal saline instead of diluted adrenaline, so as to avoid the increase of intraoperative blood pressure and increase the burden of the heart. After operation, the vagina was filled with lodophor gauze for 48 hours to prevent hematoma. The surgical procedure becomes easier and more feasible thanks to the application of mesh box, which has reduced the experience requirement on surgeons in such pelvic floor surgery. However, only after completing sufficient theoretical and technical training, and being experienced in vaginal surgery, can the surgeons perform the pelvic floor surgery. Given that TVM use has dropped precipitously in the USA due to medicolegal issues related to class action lawsuits against the manufacturers of the TVM kits, the use of TVM can be further optimized through the following aspects:First, the indications of TVM should be further refined. For patients with relatively high risk of anesthesia and surgery, TVM should be given priority to avoid re-surgery due to recurrence. In addition, 3D printing technology ${ }^{25}$ can be used when training young doctors and patients for pre-operative communication. The printout of the patient's pelvic floor structure not only allows the patient to understand the surgical procedure and risks more directly, but also allows young doctors to relearn the pelvic floor organ structure from $2 D$ to $3 D$

Our study has some limitations. We acknowledge the limited number of patients included in this study and its retrospective nature. Due to the influence of traditional concepts, the sexual activity of elderly women seems lower than other regions, and preoperative sexual life only accounts for $5.6 \%$. The limited number of patients could not fully reflect the influence of TVM on dyspareunia .In addition, not all women undergoing pelvic organ prolapse repair at our institution enrolled in the database

\section{Conclusions}

Findings of this study suggest that, TVM placement is effective on repairing POP, which can also achieve high satisfaction. Altogether it is associated with potential operation-related and mid-term complications, they can be treated through some conventional methods and attained favorable results. Therefore, we believe that it is safe to treat POP through TVM in the hands of experienced surgeons.

\section{Abbreviations}

TVM: transvaginal mesh; POP: pelvic organ prolapse; PFD: pelvic floor defect; POP-Q: pelvic organ prolapse Questionnaire; PFIQ-7: Pelvic Floor Influence Questionnaire-7; PFDI-20: Pelvic Floor Dysfunction Inventory 20; PGI-I: Patient Global Impression of Improvement; FDA: Food and Drug Administration; POPDI-6: pelvic organ prolapse distress inventory-6; CRADI-8: Colorectal-anal distress inventory-8; UDI-6: Urinary distress inventory 6

\section{Declarations}

Acknowledgements 
Not applicable

\section{Funding}

Not applicable

\section{Availability of data and materials}

Not applicable

\section{Authors Contributions}

Zeyu Chen: Project development, Data Collection, Manuscript writing

Wensheng Du: Project development

Haitao Zhu: Data collection,Project development

\section{Ethics approval and consent to participate}

This project was submitted and approved by approved by the IRB of the of Affiliated Hospital of Xuzhou Medical University under the number XYFY2019-KL093.

\section{Consent for publication}

To participate, the women signed the Free and Informed Term of Consent.

\section{Competing interests}

The authors declare that they have no competing interests

\section{References}

1. Maher C, Feiner B, Baessler K,et al. Transvaginal mesh or grafts compared with native tissue repair for vaginal prolapse.Cochrane Database Syst Rev 2016;2:CD012079.

2. Feiner, B., J.E. Jelovsek and C. Maher, Efficacy and safety of transvaginal mesh kits in the treatment of prolapse of the vaginal apex: a systematic review. BJOG, 2009. 116(1): p. 15-24.

3. Withagen MI,Milani AL, den Boon J,et al. Trocar-guided mesh compared with conventional vaginal repair in recurrent prolapse: a randomized controlled trial.Obstet Gynecol 2011;117:242-250.

4. Lee D.Management of complications of mesh surgery.Curr Opin Urol 2015;25:284-291.

5. Food and Drug Administration. Obstetrical and gynecological devices; reclassification of surgical mesh for transvaginal pelvic organ prolapse repair [Internet]. 2016. [cited 2017 Feb 11] p. 35361.Report No.:2015-33165.Available from: https://www.federalregister.gov/documents/2016/01/05/2015-33165/obstetrical-and-gynecologicaldevices-reclassification-of-surgical-mesh-for-transvaginal-pelvic 
6. Bump RC, Mattiasson A, Bo K, et al. The standardization of terminology of female pelvic organ prolapse and pelvic floor dysfunction. Am J Obstet Gynecol. 1996;175:10-17

7. Vollebregt A,Fischer K,Gietelink D.Primary surgical repair of anterior vaginal prolapse: a randomised trial comparing anatomical and functional outcome between anterior colporrhaphy and trocar-guided transobturator anterior mesh.BJOG 2011;118:1518-1527.

8. Luo DY,Yang TX.Long term Follow-up of Transvaginal Anatomical Implant of Mesh in Pelvic organ prolapse.Sci Rep 2018;8:2829

9. Chan SS,Cheung RY,Lai BP,et al.Responsiveness of the Pelvic Floor Distress Inventory and Pelvic Floor Impact Questionnaire in women undergoing treatment for pelvic floor disorders. Int Urogynecol $J$ 2013;24:213-221

10. Chan, S. S. et al. Chan SS,Cheung RY,Yiu AK,et al.Chinese validation of Pelvic Floor Distress Inventory and Pelvic Floor Impact Questionnaire.Int Urogynecol J 2011;22:1305-1312

11. Barber MD,Walters MD.Short forms of two condition-specific quality-of-life questionnaires for women with pelvic floor disorders (PFDI-20 and PFIQ-7).Am J Obstet Gynecol 2005;193:103-113

12. Srikrishna S,Robinson D.Validation of the Patient Global Impression of Improvement (PGI-I) for urogenital prolapse.Int Urogynecol J 2010;21:523-528

13. Barber MD, Spino C, Janz NK et al (2009) The minimum important diferences for the urinary scales of the pelvic foor distress inventory and pelvic foor impact questionnaire. Am J Obstet Gynecol 200(5):580e1-7

14. Morley GW, DeLancey JO. Sacrospinous ligament fixation for eversion of the vagina. Am J Obstetrics Gynecology 1988;158:872-881.

15. Shull BL. Pelvic organ prolapse: anterior, superior, and posterior vaginal segment defects. Am J Obstetrics Gynecology 1999;181(July):6-11

16. Gutman R, Maher C. Uterine-preserving POP surgery. Int Urogynecology J 2013;24:1803-1813

17. Glazener $\mathrm{CM}$,Breeman S,Elders A,et al.Mesh, graft, or standard repair for women having primary transvaginal anterior or posterior compartment prolapse surgery: two parallel-group, multicentre, randomised, controlled trials (PROSPECT).Lancet 2017;389:381-392.

18. Schimpf MO, Abed H, Sanses T, et al. Graft and Mesh Use in Transvaginal Prolapse Repair: A Systematic Review. Obstet Gyneco/ 2016 Jul;128:81-91

19. Balchandra P, Marsh F, Landon C. Perioperative outcomes and prospective patient reported outcome measures for transvaginal mesh surgery. Arch Gynecol Obstet Oct 2015;292:875-882

20. Bjelic-Radisic V, Aigmueller T, Preyer $\mathrm{O}$, et al. Vaginal prolapse surgery with transvaginal mesh: results of the Austrian registry. Int Urogynecol J Aug 2014;25:1047-1052

21. de Tayrac R, Brouziyne M, Priou G, et al. Transvaginal repair of stage III-IV cystocele using a lightweight mesh: safety and 36-month outcome. Int Urogynecol J Aug 2015;26:1147-1154

22. Heinonen $P$, Aaltonen $R$, Joronen $K$, et al. Long-term outcome after transvaginal mesh repair of pelvic organ prolapse. Int Urogynecol J July 2016;27:1069-1074 
23. Frankman EA,Alperin M,Sutkin G,et al.Mesh exposure and associated risk factors in women undergoing transvaginal prolapse repair with mesh.Obstet Gynecol Int 2013;2013:926313.

24. Kelly EC,Winick-Ng J.Surgeon Experience and Complications of Transvaginal Prolapse Mesh.Obstet Gynecol 2016;128:65-72.

25. Reiner CS,Williamson T,Winklehner T,et al.The 3D Pelvic Inclination Correction System (PICS): A universally applicable coordinate system for isovolumetric imaging measurements, tested in women with pelvic organ prolapse (POP).Comput Med Imaging Graph 2017;59:28-37.

\section{Tables}

Table 1.Patient baseline characteristics

\begin{tabular}{|c|c|c|c|c|}
\hline & Total & Avaulta $®$ & Pop-UP $®$ & $P$ value \\
\hline Patients, $n$ & 89 & 52 & 37 & \\
\hline Age,years & $65.7 \pm 8.2$ & $64.9 \pm 8.4$ & $66.7 \pm 7.9$ & 0.33 \\
\hline $\mathrm{BMI}, \mathrm{Kg} / \mathrm{m}]$ & $23.0 \pm 2.7$ & $22.8 \pm 2.5$ & $23.2 \pm 2.9$ & 0.45 \\
\hline Smoking & 3๘3.3ㅁ & $1 \square 2.0 \square \square$ & $2 \square 5.4 \square \square$ & 0.37 \\
\hline Paturition(time) & $2.2 \pm 1.1$ & $2.2 \pm 0.8$ & $2.3 \pm 1.3$ & 0.54 \\
\hline Sexually active & 5ロ5.6ロ & $3 \square 5.8 \square \square$ & $2 \square 5.4 \square \square$ & 0.94 \\
\hline Anterior prolapse & $85 \square 95.5 \% \square$ & $50 \square 96.2 \square \square$ & 35ロ94.6\%ロ & \\
\hline POP-Q stage $\geq 3$ & 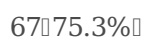 & 40ロ76.9ㅁ & $27 \square 30.3 \% \square$ & 0.67 \\
\hline Apical prolapse & $60 \square 67.4 \% \square$ & $36 \square 69.2 \square \square$ & $24 \square 27.0 \% \square$ & \\
\hline POP-Q stage $\geq 3$ & $17 \square 19.1 \% \square$ & $13 \llbracket 25.0 \% \square$ & $4 \square 7.7 \% \square$ & 0.93 \\
\hline Posterior prolapse & $36 \square 40.4 \% \square$ & $23 \llbracket 44.2 \% \square$ & 13ロ35.1\%॰ & \\
\hline POP-Q stage $\geq 3$ & $11 \square 12.4 \% \square$ & $8 \square 15.4 \% \square$ & 3๑8.1\%ロ & 0.30 \\
\hline PFDI-20 & $86.0 \pm 26.9$ & $86.9 \pm 27.0$ & $84.9 \pm 27.1$ & 0.73 \\
\hline POPDI & $42.8 \pm 15.8$ & $42.2 \pm 16.1$ & $43.7 \pm 15.7$ & 0.67 \\
\hline CRADI & $10.2 \pm 9.6$ & $10.8 \pm 9.6$ & $9.3 \pm 9.6$ & 0.47 \\
\hline UDI & $33.0 \pm 10.2$ & $33.8 \pm 10.5$ & $31.9 \pm 9.8$ & 0.37 \\
\hline PFIQ-7 & $64.4 \pm 20.9$ & $64.5 \pm 21.0$ & $64.3 \pm 20.9$ & 0.96 \\
\hline
\end{tabular}

$\mathrm{BMI}=$ body mass index

Values are presented as mean \pm SD or number of cases (and \%) in each group.

Chinese version of the Pelevic Floor Distress Inventory(PFDI-20) and Chinese validation of the Pelvic Floor Impact Questionnaire Short Form(PFIQ-7) were used to quantify the outcomes and both have the perfect score of 300 


\section{Table 2 .Operative outcomes}

\begin{tabular}{|c|c|c|c|c|}
\hline & Total & Avaulta ${ }^{\circledR}$ & Pop-Up® & $P$ value \\
\hline \multicolumn{5}{|l|}{ Surgical procedure } \\
\hline Opreating time(min) & $60.0 \pm 13.0$ & $61.1 \pm 12.6$ & $58.4 \pm 13.6$ & 0.34 \\
\hline Blood loss (mL) & $57.2 \pm 21.9$ & $60.4 \pm 26.2$ & $52.7 \pm 12.8$ & 0.07 \\
\hline Length of stay(day) & $8.4 \pm 3.5$ & $10.3 \pm 2.0$ & $7.6 \pm 2.8$ & 0.28 \\
\hline \multicolumn{5}{|l|}{ Surgical complications* } \\
\hline Bladder injury & 0 & 0 & 0 & \\
\hline Bowel injury & 0 & 0 & 0 & \\
\hline Transvaginal incision delayed healing & 3 & 2 & 1 & \\
\hline \multicolumn{5}{|l|}{ Mesh exposure } \\
\hline Urinary infaction & 3 & 1 & 2 & \\
\hline urinary incontinence & 8 & 5 & 3 & \\
\hline \multirow[t]{2}{*}{ Dyspareunia } & 2 & 1 & 1 & \\
\hline & 2 & 2 & 0 & \\
\hline
\end{tabular}

Continuous variables are expressed with mean \pm SD and quaitative variables with $n][0$

*Complications range from postoperative to last follow-up

Table 3. 2 years follow-up of patient and retreatment outcomes 


\begin{tabular}{|c|c|c|c|c|}
\hline & Total & Avaulta & Pop-Up & $P$ value \\
\hline PFDI-20 & $25.3 \pm 10.7$ & $25.7 \pm 11.1$ & $24.7 \pm 10.3$ & 0.73 \\
\hline POPDI-6 & $9.4 \pm 5.1$ & $9.9 \pm 5.0$ & $8.7 \pm 5.2$ & 0.67 \\
\hline CRADI-8 & $3.7 \pm 5.2$ & $3.5 \pm 4.6$ & $4.1 \pm 5.9$ & 0.47 \\
\hline UDI-6 & $12.2 \pm 6.7$ & $12.4 \pm 7.1$ & $11.9 \pm 6.1$ & 0.37 \\
\hline PFIQ-7 & $17.3 \pm 7.5$ & $17.8 \pm 7.6$ & $16.7 \pm 7.4$ & 0.96 \\
\hline \multirow[t]{2}{*}{ PGI-I } & $1.31 \pm 0.59$ & $1.35 \pm 0.82$ & $1.27 \pm 0.84$ & 0.53 \\
\hline & Total & Avaulta & Pop-Up & $P$ value \\
\hline$\Delta$ PFDI-20 & $60.8 \pm 24.2$ & $61.2 \pm 22.8$ & $60.1 \pm 26.2$ & 0.86 \\
\hline$\Delta$ POPDI-6 & $33.5 \pm 15.8$ & $32.3 \pm 15.4$ & $35.1 \pm 16.1$ & 0.44 \\
\hline$\Delta$ CRADI-8 & $6.5 \pm 6.2$ & $7.3 \pm 6.9$ & $5.5 \pm 4.9$ & 0.12 \\
\hline$\Delta$ UDI-6 & $20.8 \pm 10.0$ & $21.5 \pm 9.2$ & $19.5 \pm 11.1$ & 0.48 \\
\hline$\Delta$ PFIQ-7 & $47.1 \pm 20.0$ & $46.8 \pm 18.6$ & $57.6 \pm 2.0 .8$ & 0.22 \\
\hline Any retreatment & $9(10.1)$ & $6(11.5)$ & $3(6.1)$ & 0.60 \\
\hline
\end{tabular}

Continuous variables are expressed with mean \pm SD and quaitative variables with $n \rrbracket \mid 00$

Any retreatment includes midurethal sling placement, excision the exposed mesh, medication for vaginal infection, and other complication management. 